\title{
Vibrational Properties of Ge-Sb-Te Phase-Change Alloys Studied by IR and Raman Spectroscopy at Different Temperatures
}

\author{
K. Shportko and M. Wuttig
}

\begin{abstract}
Ge-Sb-Te phase-change alloys are employed as active elements in optical data storages and are promising for new generation of the electronic memory devices (Wuttig M, Nat Mater 4:265-266, 2008). Upon crystallization, their visible and infrared dielectric properties change dramatically due to the formation of resonant bonds (Shportko K, Kremers S, Woda M, Lencer D, Robertson J, Wuttig M, Nat Mater 7(8):653-658, 2008).

In this work we report on the systematic compositional dependencies of the intensities of the bands in Raman spectra of amorphous Ge-Sb-Te alloys. These dependencies correlate with evolution of concentration of the different structural units in amorphous Ge-Sb-Te alloys. Obtained compositional trends may enable one to predict vibrational properties of other amorphous $\mathrm{Ge}-\mathrm{Sb}$-Te chalcogenides. We have also studied the change of the anharmonicity upon crystallization of GeSb-Te alloys, and the influence of vacancy concentration and vacancy ordering on the anharmonicity. The temperature-dependent IR and Raman study of vibrational modes directly reveals a correlation between anharmonicity and vacancy concentration and ordering.
\end{abstract}

\section{References}

1. Wuttig M (2008) Nat Mater 4:265-266

2. Shportko K, Kremers S, Woda M, Lencer D, Robertson J, Wuttig M (2008) Nat Mater 7(8):653-658

K. Shportko $(\square)$

V.E. Lashkaryov Institute for Semiconductor Physics, National Academy of Sciences of Ukraine, Kyiv, Ukraine

e-mail: konstantin@ shportko.com; kostiantyn.shportko@ rwth-aachen.de

M. Wuttig

I. Institute of Physics (IA), RWTH University of Technology, Aachen, Germany

(C) Springer Nature B.V. 2018

B. Di Bartolo et al. (eds.), Quantum Nano-Photonics, NATO Science for Peace

and Security Series B: Physics and Biophysics,

https://doi.org/10.1007/978-94-024-1544-5_51 